\title{
Advances in antiangiogenic treatment of small-cell lung cancer
}

This article was published in the following Dove Press journal:

OncoTargets and Therapy

12 January 2017

Number of times this article has been viewed

Hongyang Lu',
Zhiming Jiang

'Zhejiang Key Laboratory of Diagnosis \& Treatment Technology on Thoracic Oncology (Lung and Esophagus), ${ }^{2}$ Department of Thoracic Medical Oncology, Zhejiang Cancer Hospital, Hangzhou, People's Republic of China
Correspondence: Hongyang Lu

Zhejiang Key Laboratory of Diagnosis \&

Treatment Technology on Thoracic

Oncology (Lung and Esophagus), Zhejiang

Cancer Hospital, No I East Banshan

Road, Gongshu District, Hangzhou

310022, People's Republic of China

Tel +8657188122094

Fax $+8657 \mid 88122508$

Email luhy@zjcc.org.cn

\begin{abstract}
Small-cell lung cancer (SCLC), a poorly differentiated neuroendocrine malignancy, has a rapid growth rate, strong aggressiveness, early metastases, and poor prognosis. Angiogenesis greatly contributes to the metastatic process of SCLC, which has a higher vascularization compared with non-small-cell lung cancer (NSCLC). SCLC might constitute an ideal malignancy for assessing new antiangiogenic drugs and therapeutic strategies. Combining bevacizumab with paclitaxel has therapeutic benefits in chemoresistant, relapsed SCLC. The cisplatin-etoposide and bevacizumab combination, as the first-line treatment for extensive-stage SCLC, can improve progression-free survival (PFS), with an acceptable toxicity profile. Ziv-aflibercept combined with topotecan is promising for platinum-refractory SCLC. Chemotherapy combined with thalidomide cannot prolong survival. Maintenance sunitinib of $37.5 \mathrm{mg} /$ day in extensive-stage SCLC patients following induction chemotherapy with platinum/etoposide improves median PFS by 1.6 months. Serum angiopoietin-2 concentrations and vascular endothelial growth factor levels correlate with poor prognosis. Bevacizumab, ziv-aflibercept, and sunitinib are worthy of further evaluation. Thalidomide, sorafenib, pomalidomide, and cediranib may not be suitable for SCLC.
\end{abstract}

Keywords: small-cell lung cancer, bevacizumab, sunitinib antiangiogenic therapy

\section{Introduction}

Small-cell lung cancer (SCLC), a malignancy with high aggressiveness and poor differentiation, shows an alarming average overall 5 -year survival rate below $10 \%{ }^{1}$ Even after robust responses to initial chemotherapy and radiation, it recurs easily, and there are no standard therapeutic strategies for relapsed SCLC. ${ }^{2}$ SCLC grows more rapidly and metastasizes at a very fast rate. Cancer angiogenesis, reflected by the number of formed microvessels, and the related modulators, are markedly associated with the metastatic potential of non-small-cell lung cancer (NSCLC) after surgery. ${ }^{3}$ Combining bevacizumab with carboplatin/paclitaxel has an acceptable toxicity profile, with significant therapeutic benefits in Chinese individuals suffering from advanced non-squamous NSCLC. ${ }^{4}$ Bevacizumab has received approval from both the American and Chinese authorities for use as a first-line therapeutic drug in advanced NSCLC.

Angiogenesis may be a very early process in vivo which is due to the imbalance between angiogenic and antiangiogenic mediators. Angiogenesis involved in multiple, overlapping pathways and mechanisms. Hypoxia in the growing tumor can stimulate angiogenesis and activate the vascular endothelial growth factor (VEGF) pathway by inducing the production of VEGFs that bind to VEGF receptors (VEGFRs). Fibroblast growth factor (FGF) and angiopoietin-2 also sustain the continuous activation of angiogenesis. The inhibitors of VEGF and VEGFR, matrix metalloproteinase, and other target genes or proteins begin to show clinical efficacy. Since angiogenetic

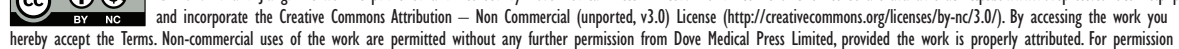
for commercial use of this work, please see paragraphs 4.2 and 5 of our Terms (https://www.dovepress.com/terms.php). 
pathways are critical to SCLC metastasis, ${ }^{5}$ SCLC may be an ideal malignancy suitable for assessing new antiangiogenic drugs and therapeutic strategies. There is no established role for antiangiogenic therapy in SCLC, and the challenge is to understand whether treatment effects in a subpopulation are lost among unselected patients. It is crucial to identify predictive biomarkers for antiangiogenic therapy. This review focuses on the advances of antiangiogenic treatment in SCLC.

\section{Bevacizumab}

Bevacizumab is a monoclonal antibody targeting VEGF; it inhibits angiogenesis in malignant tumors, including NSCLC and SCLC. It is the drug that has demonstrated an impact on overall survival (OS) in the first-line treatment and maintenance therapy for stage IV non-squamous NSCLC. ${ }^{4}$ In extensive-stage SCLC, bevacizumab is the most studied antiangiogenic drug. Topotecan is one of the main drugs used for the second-line treatment of relapsed SCLC. An open-label multicenter single-arm phase II study showed that administration of bevacizumab and oral topotecan improved the 3-month progression-free survival (PFS) (65\%) compared with the historical control (50\%), but did not meet the predefined criteria. Fifty patients were enrolled, and median PFS was 6.24 months for the sensitive subgroup and 2.91 months for the resistant subgroup. None of the patients achieved complete response, and the objective response rate (ORR) was 16\%. The topotecan-bevacizumab combination in treating relapsed SCLC was promising and needs to be further evaluated. ${ }^{6}$ Administration of bevacizumab plus irinotecan for the treatment of relapsed, resistant SCLC, for which only 32 patients were enrolled and 28 of them were eligible for evaluation, demonstrates promising efficacy and low toxicity compared to historical controls, and further investigation is warranted. ${ }^{7}$ Addition of bevacizumab to paclitaxel was found to be feasible and effective in chemoresistant, relapsed SCLC as salvage treatment, although no improvement in outcome was found in relapsed chemosensitive SCLC patients. ${ }^{8,9} \mathrm{~A}$ study assessed SCLC patients who relapsed within 3 months of first-line chemotherapy. Treatment with a combination of paclitaxel and bevacizumab for 28-day cycles showed an overall ORR of $20 \%$ and a disease control rate (DCR) of $36.7 \%$. In addition, median response duration, PFS, and OS were $2.5,2.7$, and 6.3 months, respectively. Grade 3 and 4 side effects only included neutropenia, diarrhea, and fatigue. This combination therefore represents a suitable substitute treatment, which should be imperatively evaluated in relapsed, chemoresistant SCLC patients. ${ }^{9}$ The French Cooperative Thoracic Intergroup (IFCT)-0802 study showed that bevacizumab administration following induction chemotherapy in extensive-stage SCLC does not ameliorate patient response or PFS. ${ }^{10}$ The dose of bevacizumab administered for the IFCT-0802 study was $7.5 \mathrm{mg} / \mathrm{kg}$, not the routine dose of $15 \mathrm{mg} / \mathrm{kg}$. A single-institution experience showed that cisplatin-etoposide (EP) combined with bevacizumab before oral etoposide administration and bevacizumab maintenance therapy shows likely more efficacy and is relatively safe in extensive-stage SCLC patients. ${ }^{11}$ Another randomized phase II study of the addition of bevacizumab to cisplatin or carboplatin plus etoposide (EC) for the treatment of extensive-stage SCLC achieved the similar results which only improved PFS but not OS with an acceptable toxicity profile. ${ }^{12}$ Irinotecan plus carboplatin can also be used in the treatment of extensive-stage SCLC. A phase II trial assessed the effect of irinotecan, carboplatin, and bevacizumab in the treatment of these patients. Fifty-one patients were enrolled and four patients were unassessable. ORR was $84 \%$, median time to progression (TTP) 9.13 months, and median OS 12.1 months. No significant bleeding occurred. Larger randomized trials comparing irinotecan, carboplatin, and bevacizumab with chemotherapy alone are further warranted..$^{13} \mathrm{EP}$, as a monotherapy or used in combination with bevacizumab as first-line therapy for extensive-stage SCLC, was assessed in a multicenter, phase III randomized trial. All the subjects were randomized to arm A (EP/EC) or B (same chemotherapy combined with bevacizumab). Interestingly, addition of bevacizumab improved PFS (5.7 vs 6.7 months, $P=0.030)$ with tolerable toxic effects, while there was no statistical difference in OS, the primary endpoint. ${ }^{14}$ Tracheoesophageal fistulae are rare but serious complications in lung and esophageal cancers. Medication warnings and a change in bevacizumab is labeling, due to combination of bevacizumab and chemoradiotherapy is associated with a relatively high incidence of tracheoesophageal fistulae formation in limited stage SCLC. ${ }^{15}$ Bevacizumab combined chemotherapy in SCLC can improve PFS not OS, but the evidence is weak and most of them are derived from phase II trials. Although toxicity is controllable, tracheoesophageal fistulae should be paid more attention, especially with chemoradiotherapy.

\section{Ziv-aflibercept}

Ziv-aflibercept comprises high-affinity binding domains from VEGFR1 and VEGFR2 fused to the fragment crystallizable (Fc) region of human immunoglobulin ( $\mathrm{IgG}) 1$. It recognizes placental growth factor, VEGF-A and VEGF-B, and essentially removes these ligands. ${ }^{16,17}$ It is used in the USA to treat patients with metastasized colorectal cancer. ${ }^{18}$ One hundred and eighty-nine patients were enrolled in a phase II trial, 
assessing SCLC subjects administered platinum with weekly topotecan alone and in combination with ziv-aflibercept. ${ }^{17}$ The patients were treated every 3 weeks and classified according to platinum sensitivity. Three-month PFS was markedly ameliorated by ziv-aflibercept in platinum-refractory disease unlike the sensitive type. The DCR was increased in the combination group compared with those treated with topotecan as monotherapy, regardless of the platinum sensitivity status. The OS of patients who received topotecan combined with ziv-aflibercept was similar to that of those administered topotecan. An addition of ziv-aflibercept led to more grades 3-5 toxicities. Ziv-aflibercept combined with topotecan is a promising therapeutic option for platinum-refractory SCLC. Toxicity and efficacy need to be in a balance, and a further phase III trial is warranted.

\section{Endostar}

Folkman et al first identified endostatin, the $20 \mathrm{kDa}$ internal fragment of the carboxy terminus of collagen XVIII, in 1997. Endostar, a novel recombinant human endostatin expressed and purified in Escherichia coli, is one of the most effective angiogenesis inhibitors. ${ }^{19}$ A phase II trial assessing EP and endostar for extensive-stage SCLC demonstrated slightly higher PFS and OS compared with historical controls treated with this chemotherapeutic regimen alone, with acceptable side effects. ${ }^{20}$ Addition of endostar to EC in the treatment of extensive-stage SCLC was evaluated for efficacy and safety. One hundred and forty patients with extensive-stage SCLC were randomized to EC alone or EC combined endostar, with endostar administered thereafter until intolerable toxic effects or progression. Median PFS were 6.4 and 5.9 months in the EC-endostar combination and EC groups, respectively. The ORRs of combination group and EC as monotherapy group were $75.4 \%$ and $66.7 \%$, respectively $(P=0.348)$. Median OS rates were similar for the two groups. There were no significant differences between the two groups in the incidence of both nonhematological and grade 3-4 hematological toxicities. ${ }^{21}$ An addition of endostar to EC may not be suitable for the treatment of extensive-stage SCLC as this regimen did not improve OS, PFS, and ORR.

\section{Thalidomide}

Thalidomide, though a drug prescribed to treat morning sickness in pregnant women, induced birth defects, but now it is an effective drug for cancer treatment through antiangiogenesis and potent immunomodulatory effects. Mall et al reported a long-term survival in an SCLC patient after administration of thalidomide and combination chemotherapy. ${ }^{22}$ A phase II study assessing thalidomide as maintenance therapy in extensive-stage SCLC after response to chemotherapy showed that thalidomide has acceptable side effects. ${ }^{23}$ Thalidomide combined chemotherapy for maintenance in SCLC was also assessed in 25 chemotherapy-naive SCLC patients; interestingly, chemotherapy-related toxicity was not increased, with an ORR of 68\%. Median PFS and OS were 8.3 and 10.1 months, respectively. ${ }^{24}$ A phase III trial evaluating thalidomide for advanced SCLC after chemotherapy showed that this drug did not significantly improve patient survival. However, some benefits were found for patients with a performance status of 1 or $2 .{ }^{25}$ In addition, 119 patients received two cycles of etoposide, cisplatin, cyclophosphamide, and $4^{\prime}$-epidoxorubicin (PCDE). Responders to this treatment were randomly divided for the administration of four additional PCDE cycles in combination with thalidomide and placebo, respectively. After two treatment cycles, an ORR of $81.5 \%$ was obtained, with 92 individuals randomly divided into placebo $(n=43)$ and thalidomide $(n=49)$ groups. ${ }^{25}$ Another study assessing thalidomide/chemotherapy for antiangiogenic effects in SCLC also demonstrated that combining thalidomide with chemotherapy showed no improvement in survival but promoted the occurrence of thrombotic events. ${ }^{26}$ Several known inflammatory and angiogenic markers were assessed, and none were specific to patients who benefited from thalidomide treatment. ${ }^{27}$ Patients with a performance status of 1 or 2 may be suitable to use thalidomide, but it needs a prospective randomized controlled study to verify.

\section{Sunitinib}

Sunitinib (SU11248) shows a direct anticancer effect via KIT (CD117) and inhibits angiogenesis through VEGFR, and platelet-derived growth factor receptor- $\beta$ (PDGFR- $\beta$ ). ${ }^{28}$ A phase II clinical trial, in which the treatment regimen consisted of a 6-week cycle of sunitinib ( $50 \mathrm{mg}$ daily for 4 weeks followed by 2 weeks off) in relapsed or refractory SCLC, showed that the majority of subjects tolerated the therapy, with short median PFS. With the currently used regimen, further assessment may not be useful for sunitinib. ${ }^{29}$ Nine patients were registered in a single-arm phase II study of sunitinib, with a loading dose of $150 \mathrm{mg}$ followed by $37.5 \mathrm{mg} /$ day, in patients with SCLC who were either chemo naive (extensive disease) or have a "sensitive" relapse. DCR at 8 weeks was achieved in two patients, and one toxic-related death was reported due to bronchial hemorrhage. The study was closed because of low accrual, with only 9 of required 48 patients accrued. ${ }^{30}$ Cancer and Leukemia Group B (CALGB) (Alliance) 30504 is a phase II trial, which evaluated sunitinib vs placebo for maintenance in individuals suffering from extensive-stage 
SCLC following induction chemotherapy with platinum/ etoposide. ${ }^{31}$ Patients without progression were assigned to sunitinib and placebo groups and treated until progression. One hundred and forty-four SCLC patients were enrolled, of which 138 were subjected to chemotherapy. A total of 95 subjects were randomized, among which 85 were administered maintenance treatment with sunitinib $(n=44)$ or placebo $(\mathrm{n}=41)$. Most prominent grade 3 adverse events (AEs) were fatigue (19\%), leukopenia (7\%), reduced neutrophil counts (14\%), and reduced platelet count (7\%) in the sunitinib group; fatigue $(10 \%)$ was also found as $\mathrm{AE}$ in the placebo group. Grade 4 AEs include pancreatitis, hypocalcemia, elevated lipase (in the same individual), and gastrointestinal hemorrhage $(n=1)$ in sunitinib-treated patients; hypernatremia $(n=1)$ and thrombocytopenia $(n=1)$ were found in the placebo group. Median PFS in maintenance therapy were 3.7 and 2.1 months, respectively, for the treatment and placebo groups, respectively; median OS were similar in the two groups. ${ }^{31} \mathrm{~A}$ trend toward PFS and OS improvement was found in individuals treated with prophylactic cranial irradiation and sunitinib. ${ }^{32}$ This should not alter the current standard of care as only a small absolute improvement in median PFS (1.6 months) was observed over placebo; ${ }^{33,34}$ however, we believe that maintenance sunitinib of $37.5 \mathrm{mg} /$ day met its primary endpoint with an improvement in median PFS of 1.6 months over placebo and toxicity included grade 3 fatigue in 19\%. Maintenance sunitinib is safe and should be further evaluated in phase III studies. Sunitinib maintenance following irinotecan and carboplatin as the first-line treatment for patients with extensivestage SCLC was evaluated in a phase II study. Patients with stable disease or responding disease proceeded to sunitinib monotherapy ( $25 \mathrm{mg}$ orally daily) until disease progression or unacceptable toxicity. Thirty-four patients were enrolled, of which 17 were initiated with sunitinib monotherapy. Oneyear OS was 54\%, and median TTP was 7.6 months. Grade $3 / 4$ toxicity was rare during sunitinib monotherapy. ${ }^{35}$ Further study of sunitinib therapy in a randomized phase III trial maintenance would be appropriate. Improving the outcome of patients with extensive-stage SCLC following induction chemotherapy might need prophylactic cranial irradiation and thoracic radiotherapy, as well as maintenance systemic treatment. $^{32}$

\section{Sorafenib}

Sorafenib is a multi-tyrosine kinase inhibitor (TKI) targeting Raf-kinase, PDGFR, and VEGFR. The effect of EP plus concurrent and sequential sorafenib on untreated extensivestage SCLC was evaluated. ${ }^{36}$ The patients received four cycles of EP chemotherapy concurrently with sorafenib. Those without progression after four cycles were further administered a higher sorafenib dose as maintenance for $\leq 12$ months. The regimen of EP combined sorafenib at current dose levels has significant toxicity, and the efficacy results have been disappointing. ${ }^{36}$ The suitable dose of sorafenib combined EP is still unknown, and toxicity should be paid more attention.

\section{Pomalidomide}

Pomalidomide has been shown to have potent antiangiogenic and tumoricidal effects as they inhibit proangiogenic factors, VEGF, and basic FGF. The maximum-tolerated dose (MTD), safety, and clinical benefit of pomalidomide combined with EP chemotherapy in treatment-naive patients with extensive-stage SCLC were evaluated in a phase I/IIA study. Twenty-two patients received one or more doses of the study medication. Pomalidomide at the MTD of $4 \mathrm{mg} /$ day plus standard EP seems safe, but the addition of pomalidomide does not seem to improve the therapeutic index based on the ORR (31.8\%) and median OS (49.6 weeks). ${ }^{37}$ Pomalidomide may be not a promising drug in SCLC.

\section{Cediranib}

Cediranib, an oral TKI of VEGFR-1, VEGFR-2, VEGFR-3, and c-kit, has antitumor activity in recurrent epithelial ovarian cancer, fallopian tube cancer, and peritoneal cancer with predictable toxicities. ${ }^{38}$ A phase II trial assessing the safety and efficacy of cediranib was carried out in refractory or recurrent SCLC. A dose of $45 \mathrm{mg}$ of cediranib was employed daily (12 patients), and later this was decreased to $30 \mathrm{mg}$ daily for subsequent individuals due to intolerance. Of the twenty-five patients enrolled, nine showed stable disease, and none had partial response. Median PFS and OS were 2 and 6 months, respectively. Cediranib treatment resulted in no objective response. ${ }^{39}$ Cediranib is not worth for further study in SCLC.

\section{Vandetanib}

Vandetanib is an inhibitor of VEGFR and epidermal growth factor receptor (EGFR). Vandetanib (300 mg/day) or matched placebo maintenance therapy in SCLC patients responding to induction chemotherapy was evaluated in a double-blind randomized phase II trial. One hundred and seven patients were accrued, and the results revealed that vandetanib had more toxicity, including asymptomatic corrected QT interval prolongation, and required more dose modifications for rash and gastrointestinal toxicity. Vandetanib failed 
to demonstrate efficacy as maintenance therapy in these patients. ${ }^{40}$ Another randomized, double-blind, phase II trial of platinum therapy plus etoposide with or without concurrent vandetanib (100 mg daily) in patients with previously untreated SCLC for up to total four cycles showed that the addition of vandetanib did not improve outcomes, and that nonhematologic toxicity increased; the trial included 74 patients of which 33 were randomized to each arm. ${ }^{41}$ Vandetanib may not be a promising drug in SCLC.

\section{Nintedanib}

Nintedanib, a potent, oral angiokinase inhibitor that targets the proangiogenic pathways mediated by VEGFR1-3, FGF receptor (FGFR) 1-3, and PDGFR- $\alpha$ and $\beta$, has demonstrated a survival benefit in combination with docetaxel vs docetaxel alone in previously treated lung adenocarcinoma. A phase II study was conducted to evaluate the efficacy and safety of nintedanib with $200 \mathrm{mg}$ BID daily in a 4-week cycle until progression or intolerable toxicity in relapsed SCLC patients. Twenty-four patients were enrolled, who exhibited progression after one or two prior chemotherapy or chemo/radiotherapy, of which 22 patients were evaluable for response. Nintedanib exhibited only limited activity and showed the following results: ORR 5\%, median PFS 1.0 month, and OS 9.8 months with grade 3 hepatic enzyme elevation occurring at $23 \% .^{42}$

\section{Prognostic markers}

VEGF is critical to the angiogenic process, and its circulating levels could be a further prognostic marker in SCLC. Angiopoietin-2 is an angiogenesis-related cytokine. ${ }^{43}$ Ustuner et $\mathrm{al}^{44}$ reported that reduced circulating levels of VEGF represent an independent prognostic factor for SCLC. Investigation of VEGF and its related receptors for predicting the chemotherapeutic response seems worthless. ${ }^{44}$ Research by Lucchi showed that microvessel amounts and VEGF expression markedly affect patient survival, and constitute independent prognostic factors. ${ }^{3}$ Serum VEGF and soluble VEGFR titers failed to identify predictive biomarkers. High baseline circulating angiopoietin-2 levels are correlated with worse OS and constitute an independent predictor of OS. ${ }^{45}$

\section{Conclusion}

None of the antiangiogenic drugs were approved in the treatment of SCLC because the data were not sufficient and satisfactory, despite some drugs were promising. The use of angiogenesis inhibitors in extensive SCLC is still restricted. Bevacizumab combined with platinum/etoposide for extensive-stage SCLC can improve PFS and requires further studies. Maintenance sunitinib of $37.5 \mathrm{mg} /$ day in extensive-stage SCLC is safe and deserves further evaluation in phase III studies. Ziv-aflibercept combined with topotecan is promising for platinum-refractory SCLC. Chemotherapy combined with thalidomide, sorafenib, or pomalidomide may not be suitable for SCLC treatment. Cediranib failed to be effective in recurrent or refractory SCLC. Serum VEGF and angiopoietin-2 levels were both correlated with poor prognosis.

\section{Acknowledgments}

The present study was funded by the Zhejiang Provincial Natural Science Foundation of China (No LY15H290001), the Public Welfare Technology Application Studies Program of Zhejiang Province (No 2016C33118), and the 1022 Talent Training Program of Zhejiang Cancer Hospital.

\section{Disclosure}

The authors report no conflicts of interest in this work.

\section{References}

1. Schneider BJ, Kalemkerian GP. Personalized therapy of small cell lung cancer. Adv Exp Med Biol. 2016;890:149-174.

2. Minami T, Kijima T, Kohmo S, et al. Overcoming chemoresistance of small-cell lung cancer through stepwise HER2-targeted antibodydependent cell-mediated cytotoxicity and VEGF-targeted antiangiogenesis. Sci Rep. 2013;3:2669.

3. Fontanini G, Lucchi M, Vignati S, et al. Angiogenesis as a prognostic indicator of survival in non-small-cell lung carcinoma: a prospective study. J Natl Cancer Inst. 1997;89(12):881-886.

4. Zhou C, Wu YL, Chen G, et al. BEYOND: a randomized, doubleblind, placebo-controlled, multicenter, phase III study of first-line carboplatin/paclitaxel plus bevacizumab or placebo in chinese patients with advanced or recurrent nonsquamous non-small-cell lung cancer. J Clin Oncol. 2015;33(19):2197-2204.

5. Lucchi M, Mussi A, Fontanini G, Faviana P, Ribechini A, Angeletti CA. Small cell lung carcinoma (SCLC): the angiogenic phenomenon. Eur J Cardiothorac Surg. 2002;21(6):1105-1110.

6. Spigel DR, Waterhouse DM, Lane S, Legenne P, Bhatt K. Efficacy and safety of oral topotecan and bevacizumab combination as second-line treatment for relapsed small-cell lung cancer: an open-label multicenter single-arm phase II study. Clin Lung Cancer. 2013;14(4):356-363.

7. Trafalis DT, Alifieris C, Stathopoulos GP, Sitaras N. Phase II study of bevacizumab plus irinotecan on the treatment of relapsed resistant small cell lung cancer. Cancer Chemother Pharmacol. 2016;77(4):713-722.

8. Jalal S, Bedano P, Einhorn L, et al. Paclitaxel plus bevacizumab in patients with chemosensitive relapsed small cell lung cancer: a safety, feasibility, and efficacy study from the Hoosier Oncology Group. J Thorac Oncol. 2010;5(12):2008-2011.

9. Mountzios G, Emmanouilidis C, Vardakis N, et al. Paclitaxel plus bevacizumab in patients with chemoresistant relapsed small cell lung cancer as salvage treatment: a phase II multicenter study of the Hellenic Oncology Research Group. Lung Cancer. 2012;77(1):146-150.

10. Pujol JL, Lavole A, Quoix E, et al; French Cooperative Thoracic Intergroup (IFCT); French Cooperative Thoracic Intergroup IFCT. Randomized phase II-III study of bevacizumab in combination with chemotherapy in previously untreated extensive small-cell lung cancer: results from the IFCT-0802 trial. Ann Oncol. 2015;26(5):908-914. 
11. Petrioli R, Roviello G, Laera L, et al. Cisplatin, etoposide, and bevacizumab regimen followed by oral etoposide and bevacizumab maintenance treatment in patients with extensive-stage small cell lung cancer: a single-institution experience. Clin Lung Cancer. 2015; 16(6):e229-e234.

12. Spigel DR, Townley PM, Waterhouse DM, et al. Randomized phase II study of bevacizumab in combination with chemotherapy in previously untreated extensive-stage small-cell lung cancer: results from the SALUTE trial. J Clin Oncol. 2011;29(16):2215-2222.

13. Spigel DR, Greco FA, Zubkus JD, et al. Phase II trial of irinotecan, carboplatin, and bevacizumab in the treatment of patients with extensive-stage small-cell lung cancer. J Thorac Oncol. 2009;4(12):1555-1560.

14. Tiseo M, Boni L, Ambrosio F, et al. Italian multicenter phase III randomized study of cisplatin-etoposide with or without bevacizumab as first-line treatment in extensive stage small cell lung cancer (SCLC): GOIRC-AIFA FARM6PMFJM trial. J Clin Oncol. 2016;34(Suppl): abstr 8513.

15. Spigel DR, Hainsworth JD, Yardley DA, et al. Tracheoesophageal fistula formation in patients with lung cancer treated with chemoradiation and bevacizumab. J Clin Oncol. 2010;28(1):43-48.

16. Kim ES, Serur A, Huang J, et al. Potent VEGF blockade causes regression of coopted vessels in a model of neuroblastoma. Proc Natl Acad Sci U S A. 2002;99(17):11399-11404.

17. Allen JW, Moon J, Redman M, et al. Southwest Oncology Group S0802: a randomized, phase II trial of weekly topotecan with and without zivaflibercept in patients with platinum-treated small-cell lung cancer. J Clin Oncol. 2014;32(23):2463-2470.

18. Van Cutsem E, Tabernero J, Lakomy R, et al. Addition of aflibercept to fluorouracil, leucovorin, and irinotecan improves survival in a phase III randomized trial in patients with metastatic colorectal cancer previously treated with an oxaliplatin-based regimen. J Clin Oncol. 2012; 30(28):3499-3506.

19. O'Reilly MS, Boehm T, Shing Y, et al. Endostatin: an endogenous inhibitor of angiogenesis and tumor growth. Cell. 1997;88(2):277-285.

20. Zhou ZT, Zhou FX, Wei Q, Zou LY, Qin BF, Peng XS. Phase II study of cisplatin/etoposide and endostar for extensive-stage small-cell lung cancer. Cancer Chemother Pharmacol. 2011;68(4):1027-1032.

21. Lu S, Li L, Luo Y, et al. A multicenter, open-label, randomized phase II controlled study of rh-endostatin (Endostar) in combination with chemotherapy in previously untreated extensive-stage small-cell lung cancer. J Thorac Oncol. 2015;10(1):206-211.

22. Mall JW, Philipp AW, Mall W, Pollmann C. Long-term survival of a patient with small-cell lung cancer (SCLC) following treatment with thalidomide and combination chemotherapy. Angiogenesis. 2002;5(1-2):11-13.

23. Dowlati A, Subbiah S, Cooney M, et al. Phase II trial of thalidomide as maintenance therapy for extensive stage small cell lung cancer after response to chemotherapy. Lung Cancer. 2007;56(3):377-381.

24. Lee SM, James L, Buchler T, Snee M, Ellis P, Hackshaw A. Phase II trial of thalidomide with chemotherapy and as maintenance therapy for patients with poor prognosis small-cell lung cancer. Lung Cancer. 2008;59(3):364-368.

25. Pujol JL, Breton JL, Gervais R, et al. Phase III double-blind, placebocontrolled study of thalidomide in extensive-disease small-cell lung cancer after response to chemotherapy: an intergroup study FNCLCC cleo04 IFCT 00-01. J Clin Oncol. 2007;25(25):3945-3951.

26. Lee SM, Woll PJ, Rudd R, et al. Anti-angiogenic therapy using thalidomide combined with chemotherapy in small cell lung cancer: a randomized, double-blind, placebo-controlled trial. J Natl Cancer Inst. 2009; 101(15):1049-1057.

27. Young RJ, Tin AW, Brown NJ, Jitlal M, Lee SM, Woll PJ. Analysis of circulating angiogenic biomarkers from patients in two phase III trials in lung cancer of chemotherapy alone or chemotherapy and thalidomide. Br J Cancer. 2012;106(6):1153-1159.

28. Abrams TJ, Lee LB, Murray LJ, Pryer NK, Cherrington JM. SU11248 inhibits KIT and platelet-derived growth factor receptor beta in preclinical models of human small cell lung cancer. Mol Cancer Ther. 2003; 2(5):471-478
29. Han JY, Kim HY, Lim KY, et al. A phase II study of sunitinib in patients with relapsed or refractory small cell lung cancer. Lung Cancer. 2013;79(2):137-142.

30. Abdelraouf F, Smit E, Hasan B, et al. Sunitinib (SU11248) in patients with chemo naive extensive small cell lung cancer or who have a 'chemosensitive' relapse: a single-arm phase II study (EORTC-08061). Eur J Cancer. 2016;54:35-39.

31. Ready NE, Pang HH, Gu L, et al. Chemotherapy with or without maintenance sunitinib for untreated extensive-stage small-cell lung cancer: a randomized, double-blind, placebo-controlled Phase II StudyCALGB 30504 (Alliance). J Clin Oncol. 2015;33(15):1660-1665.

32. Salama JK, Gu L, Wang X, et al. Positive interaction between prophylactic cranial irradiation and maintenance sunitinib for untreated extensivestage small cell lung cancer patients after standard chemotherapy: a secondary analysis of CALGB 30504 (ALLIANCE). J Thorac Oncol. 2016;11(3):361-369.

33. Schneider BJ. Maintenance sunitinib for extensive-stage small cell lung cancer: a new standard, an option or a step in the right direction? Transl Lung Cancer Res. 2015;4(5):635-638.

34. Di Maio M, Bironzo P, Scagliotti GV. Raising the bar for enthusiasm when looking at results of randomized phase II trials-the case of sunitinib in small-cell lung cancer. Transl Lung Cancer Res. 2016;5(1):89-91.

35. Spigel DR, Greco FA, Rubin MS, et al. Phase II study of maintenance sunitinib following irinotecan and carboplatin as first-line treatment for patients with extensive-stage small-cell lung cancer. Lung Cancer. 2012;77(2):359-364.

36. Sharma N, Pennell N, Nickolich M, et al. Phase II trial of sorafenib in conjunction with chemotherapy and as maintenance therapy in extensivestage small cell lung cancer. Invest New Drugs. 2014;32(2):362-368.

37. Ellis PM, Jungnelius U, Zhang J, Fandi A, Beck R, Shepherd F. A phase I study of pomalidomide (CC-4047) in combination with cisplatin and etoposide in patients with extensive-stage small-cell lung cancer. $J$ Thorac Oncol. 2013;8(4):423-428.

38. Matulonis UA, Berlin S, Ivy P, et al. Cediranib, an oral inhibitor of vascular endothelial growth factor receptor kinases, is an active drug in recurrent epithelial ovarian, fallopian tube, and peritoneal cancer. J Clin Oncol. 2009;27(33):5601-5606.

39. Ramalingam SS, Belani CP, Mack PC, et al. Phase II study of Cediranib (AZD 2171), an inhibitor of the vascular endothelial growth factor receptor, for second-line therapy of small cell lung cancer (National Cancer Institute \#7097). J Thorac Oncol. 2010;5(8):1279-1284.

40. Arnold AM, Seymour L, Smylie M, et al; National Cancer Institute of Canada Clinical Trials Group Study BR.20. Phase II study of vandetanib or placebo in small-cell lung cancer patients after complete or partial response to induction chemotherapy with or without radiation therapy: National Cancer Institute of Canada Clinical Trials Group Study BR.20. J Clin Oncol. 2007;25(27):4278-4284.

41. Sanborn RE, Patel JD, Masters GA, et al. A randomized, doubleblind, phase 2 trial of platinum therapy plus etoposide with or without concurrent vandetanib (ZD6474) in patients with previously untreated extensive-stage small cell lung cancer: Hoosier Cancer Research Network LUN06-113. Cancer. Epub 2016 Sep 1; doi:10.1002/cncr.30287.

42. Han JY, Kim HY, Lim KY, Hwangbo B, Lee JS. A phase II study of nintedanib in patients with relapsed small cell lung cancer. Lung Cancer. 2016;96:108-112.

43. Mall JW, Schwenk W, Philipp AW, et al. Serum vascular endothelial growth factor levels correlate better with tumour stage in small cell lung cancer than albumin, neuron-specific enolase or lactate dehydrogenase. Respirology. 2002;7(2):99-102.

44. Ustuner Z, Saip P, Yasasever V, et al. Prognostic and predictive value of vascular endothelial growth factor and its soluble receptors, VEGFR-1 and VEGFR-2 levels in the sera of small cell lung cancer patients. Med Oncol. 2008;25(4):394-399.

45. Cañadas I, Taus Á, Villanueva X, et al. Angiopoietin-2 is a negative prognostic marker in small cell lung cancer. Lung Cancer. 2015;90(2): 302-306. 
OncoTargets and Therapy

\section{Publish your work in this journal}

OncoTargets and Therapy is an international, peer-reviewed, open access journal focusing on the pathological basis of all cancers, potential targets for therapy and treatment protocols employed to improve the management of cancer patients. The journal also focuses on the impact of management programs and new therapeutic agents and protocols on

patient perspectives such as quality of life, adherence and satisfaction. The manuscript management system is completely online and includes a very quick and fair peer-review system, which is all easy to use. Visit http://www.dovepress.com/testimonials.php to read real quotes from published authors.

Submit your manuscript here: http://www.dovepress.com/oncotargets-and-therapy-journal 